\title{
A Novel Approach for Determining the Electromagnetic Properties of a Colloidal Fluid with Magnetic Nanoparticles for Hyperthermia Applications
}

\author{
Danilo Brizi, Student Member, IEEE, Nunzia Fontana, Giulio Giovannetti, Alessandra Flori,
} Luca Menichetti, Saer Doumett, Giovanni Baldi, Agostino Monorchio, Fellow, IEEE

Analytical characterization of magnetic nanoparticles based on SAR experimental measurements.

Experimental SAR measurements from magnetic nanoparticles were acquired for different radiation conditions
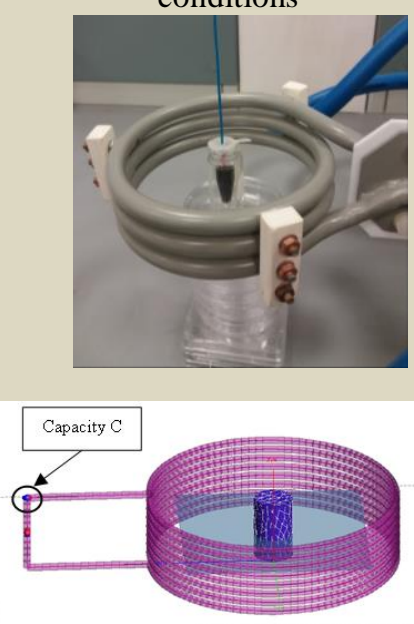
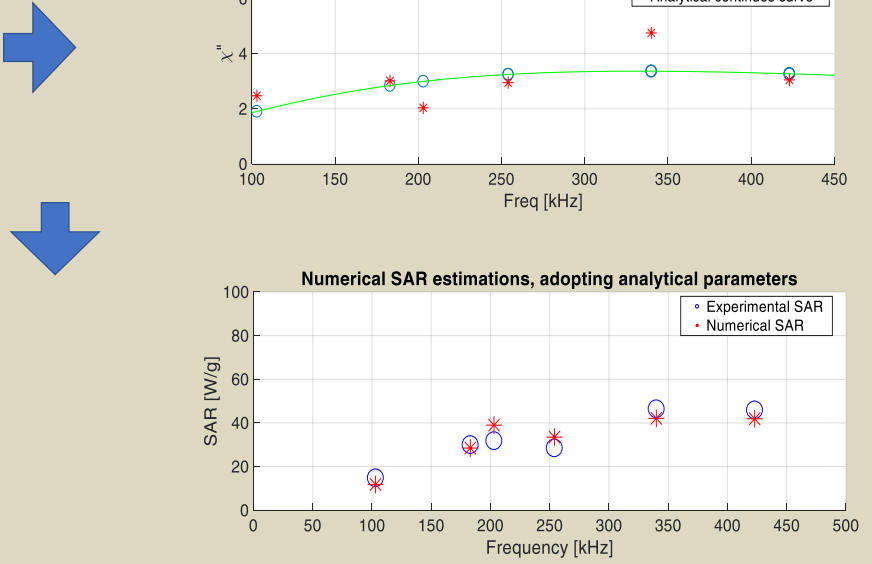

Comparison of the acquired SAR measurements with the respective numerical EM simulation using the extracted magnetic properties confirmed the validity of the analytical approach

\section{Take-Home Messages}

- A novel approach able to deeply optimize clinical treatments of magnetic hyperthermia with nanoparticles.

- Innovative determination of the complex magnetic permeability of magnetic colloidal fluid by simply using in vitro SAR measurements.

- A rapid, alternative and broadband approach for determining the electromagnetic properties of magnetic nanoparticles.

- Possibility to test the efficiency of nanoparticles in a tissue-like environment: optimized and realistic treatment planning for magnetic hyperthermia. 


\title{
A Novel Approach for Determining the Electromagnetic Properties of a Colloidal Fluid with Magnetic Nanoparticles for Hyperthermia Applications
}

\author{
Danilo Brizi, Student Member, IEEE, Nunzia Fontana, Giulio Giovannetti, Alessandra Flori, \\ Luca Menichetti, Saer Doumett, Giovanni Baldi, Agostino Monorchio, Fellow, IEEE
}

\begin{abstract}
The paper presents a general analytical method for evaluating the magnetic properties of colloidal fluid with magnetic nanoparticles and agar through in vitro Specific Absorption Rate (SAR) measurements. The approach for the determination of magnetic complex susceptibility herein presented reveals itself as simple, rapid, broadband and accurate enough to compete with alternative conventional direct methods requiring complex and expensive instrumentation. In particular, it makes use of indirect equations based on the single order Debye model (Linear Response Theory, LRT) combined with a punctual set of in vitro SAR measurements. The procedure is effective inside the range of validity of the LRT theory and it can be easily applied in the upgrowing field of magnetic hyperthermia studies.
\end{abstract}

Keywords — characterization, hyperthermia, magnetic permeability, nanomaterial, magnetic nanoparticles, oncothermia

\section{INTRODUCTION}

$\mathrm{T}$ HE CHARACTERIZATION of the magnetic properties of a colloidal magnetic fluid is fundamental for a number of applications, especially in the biomedical field: magnetic particle imaging, bio-sensing, drug delivery and magnetic hyperthermia are some of the most promising techniques [1]-[6]. In particular, an optimal characterization allows the determination of the best operative condition to convert electromagnetic energy at radiofrequency into heat at a nanoscale level, which is the main goal in magnetic hyperthermia with nanoparticles. Magnetic hyperthermia is a rising cancer therapy [7]-[10], which exploits the interactions between nanoparticles and a Radio Frequency (RF) magnetic field in order to produce a uniform and deep heating of pathological tissues in a completely non-invasive way. It is well recognized that hyperthermia induces cellular apoptosis. Apoptosis damages the functions of different important proteins that are involved in cell growth and differentiation. This leads finally to death of pathological cells. The combination with other emerging thermal therapies, such as oncothermia [11], [12], could

A. Monorchio and D. Brizi are with the Department of Information Engineering, University of Pisa, Pisa, Italy (e-mail: agostino.monorchio@unipi.it, danilo.brizi@ing.unipi.it).

N. Fontana is with Consorzio Nazionale Interuniversitario per le Telecomunicazioni (CNIT), Pisa, Italy (e-mail: nunzia.fontana@cnit.it).

G. Giovannetti and L. Menichetti are with CNR Institute of Clinical Physiology, Pisa, Italy.

A. Flori is with Fondazione CNR-Regione Toscana G.Monasterio, Pisa, Italy.

D. Saer and G. Baldi are with Centro Ricerche Colorobbia, via Pietramarina 53, Sovigliana Vinci 50053, (FI), Italy). lead to completely revolutionary cancer treatments, totally avoiding the use of more harmful therapies such as chemotherapy and radiotherapy. Moreover, it was also demonstrated that hyperthermia can enhance the efficiency of simultaneously treatments of chemotherapy and radiotherapy [13], [14].

In particular, the heat release depends on the imaginary component (loss component) of the complex magnetic susceptibility of the nanoparticles. Indeed, this aspect is of crucial relevance: the selection of the frequency, which corresponds to the highest value of the imaginary component, could lead to the greatest heat release (maximum of the Specific Absorption Rate, SAR). Consequently, it could be possible to improve significantly the efficiency of the treatment at a cellular level [15].

The Linear Response Theory (LRT), reported in [16], describes the heating of magnetic fluid exposed to an RF magnetic field. Any single nanoparticle can be represented as a magnetic dipole, which undergoes to a Debye single order relaxation phenomenon. This theory can be applied for low amplitude magnetic field and for a limited range of frequencies. Nevertheless, such conditions are typical for common magnetic hyperthermia applications. Nowadays, great efforts are directed in order to produce novel nanoassemblies, organized such as nanoclusters, in order to deeply and positively modify the relaxation behavior of the magnetic nanoparticles [15],[17].

Despite the relative simple mathematical modeling of the phenomenon, to the best of our knowledge, there is a lack in literature regarding magnetic susceptibility estimations in an environment able to mimic the real one of a clinical 
application of magnetic hyperthermia. The ideal characteristics of the experimental set-up must present clinical exposure conditions for magnetic field (in particular, frequency in the range of hundreds of $\mathrm{kHz}$ ), concentration of nanoparticles typically deliverable in a clinical set-up (few tens of $\mathrm{mg} / \mathrm{mL}$ ) and a medium able to represent a biological environment with a certain degree of fidelity. Several works addressed the problem of the direct magnetic susceptibility measurements over a wide range of frequency, typically starting from values far away from the conditions of magnetic hyperthermia (from $\mathrm{MHz}$ to $\mathrm{GHz}$ ). Commonly, such measurements were carried out with coaxial cable configurations [18]-[22]. On the other hand, papers that described direct measurements of the complex susceptibility at very low frequencies exploit very expensive instrumentation (i.e. susceptometers) [23], [24]. Besides, AC magnetometers allow to measure SAR released by the nanoparticles under the exposition to a radiofrequency magnetic field in a quite large frequency band [25]-[27]; however, no particularly realistic assumption was given to the medium of the suspension.

In order to overcome these limits, we propose a general analytical method to determine the magnetic properties of a colloidal magnetic fluid with nanoparticles and agar, starting from in vitro Specific Absorption Rate (SAR) measurements. As a matter of fact, it is very simple to obtain SAR measurements instead of direct probing the complex magnetic susceptibility. More importantly, our preliminary work considers a quite realistic environment in order to simulate a clinical treatment, using a sample made of a mixture of water, agar and nanoparticles with a clinically reasonable concentration. It could be also possible to set the properties of the experimental sample in order to mimic the properties of a specific tissue, in terms of density, viscosity and conductivities. This can be achieved by adjusting the agar quantity and the conductivity of the sample, as reported in [29]. Our approach uses indirect equations based on the single order Debye model for magnetic complex susceptibility under low field hypothesis, in a frequency range typical of magnetic hyperthermia [16], [31]. In this operative condition, the two dominant relaxation phenomena of the nanoparticles are Brownian and Néel ones [32]. Indeed, it is experimentally demonstrated in [15] and [31] that magneto-fluid's SAR release follows Rosensweig's equation under low field hypothesis for different type of nanoparticles.

In our study, we processed, at a first stage, experimental data of SAR measurements obtained by exposing a magnetic fluid to a RF magnetic field at different operative conditions (amplitude and frequency). Therefore, we analytically extracted the magnetic permeability values. Afterwards, we reported the magnetic permeability values versus the frequency. Finally, in order to validate our analytical approach, we replicated the experimental set-up through the commercial electromagnetic simulation software Feko, based on the Method of Moments (MoM).
In particular, we used the magnetic properties previously extracted through our analytical procedure and we compared the numerical SAR results with the SAR measurements, obtaining a good agreement.

Hence, the proposed method can allow an alternative experimental set-up planning of magnetic hyperthermia treatments, exploiting quick SAR measurements in vitro and an electromagnetic simulation software, which are nowadays reliable and accurate enough.

The paper is organized as follows: section II describes the theory and the analytical models used to extract magnetic properties of nanoparticles; section III reports the analytical procedure and its validation through numerical simulation; in section IV, conclusions and further developments are proposed.

\section{MethodS AND PROCEDURES}

\section{A. Behavioral modeling of magnetic nanoparticles}

We can assume the validity of the first order Debye model for the complex magnetic susceptibility of a colloidal fluid, under the assumption that the experimental conditions are characterized by a low amplitude of the magnetic field [31]. As reported in [16], the complex magnetic susceptibility can be expressed as follows:

$$
\begin{gathered}
\chi(f)=\chi^{\prime}(f)-j \chi^{\prime \prime}(f) \\
\chi^{\prime}(f)=\frac{\chi_{0}}{1+(2 \pi f \tau)^{2}} \\
\chi^{\prime \prime}(f)=\chi_{0} \frac{2 \pi f \tau}{1+(2 \pi f \tau)^{2}}
\end{gathered}
$$

where $\chi_{0}$ is the equilibrium susceptibility value and $\tau(s)$ is the effective relaxation time of the fluid, obtained as a superposition of Brownian and Néel relaxation times ( $\tau_{B}$ and $\tau_{N}$, respectively). The Brownian relaxation involves movement of the entire nanoparticle in the solution whereas Néel relaxation concerns the interaction of the RF magnetic field with the magnetic dipole that represents each single nanoparticle. These two physical phenomena are predominant under low field amplitude conditions. In the present paper, the attention is directed to the frequency range typical of magnetic hyperthermia (hundreds of $\mathrm{kHz}$ ). The magnetic nanoparticles normally exhibit another susceptibility peak at higher frequencies ( $\mathrm{GHz}$ range) due to ferromagnetic resonance [32]. However, the last physical phenomenon does not affect the magnetic susceptibility in our analyzed frequency range, thus it will be not included in our model.

The Brownian relaxation time can be expressed as [24],[33]:

$$
\tau_{B}=\frac{3 V_{H} \eta}{k_{B} T}
$$

where $V_{H}\left(\mathrm{~m}^{3}\right)$ is the hydrodynamic volume of the nanoparticles, $\eta(\mathrm{Pa} \mathrm{s})$ is the sample viscosity, $k_{B}\left(\mathrm{~m}^{2} \mathrm{~kg} \mathrm{~s}^{-2}\right.$ $\left.\mathrm{K}^{-1}\right)$ is the Boltzmann constant and $T(\mathrm{~K})$ the temperature of 
the sample. It must be noted that $V_{H}$ can be different from the actual shape and size of the single nanoparticle, due to the non-specific adsorption of the carrying medium.

The following equation describes the Néel relaxation time [24]:

$$
\tau_{N}=\tau_{0} e^{\frac{K V_{C}}{k_{B} T}}
$$

where $\tau_{0}(\mathrm{~s})$ is the attempt time, $K\left(\mathrm{~J} \mathrm{~m}^{-3}\right)$ is the anisotropy constant of the nanoparticles and $V_{C}\left(\mathrm{~m}^{3}\right)$ is the magnetic volume of the nanoparticle's core.

Finally, the effective relaxation time $\tau$ is obtained as:

$$
\tau=\left(\frac{1}{\tau_{B}}+\frac{1}{\tau_{N}}\right)^{-1}
$$

As described in [16], we can theoretically assume the validity of the Rosensweig's equation for the SAR release $(\mathrm{W} / \mathrm{kg}$ ), always under low field amplitude hypothesis:

$$
S A R=\frac{\mu_{0} \pi \chi^{\prime \prime} f H_{0}^{2}}{\phi \rho_{\mathrm{Fe}_{3} \mathrm{O}_{4}}}
$$

where $\mu_{0}(\mathrm{H} / \mathrm{m})$ is the vacuum magnetic permeability, $\chi^{\prime \prime}$ is the imaginary component of complex magnetic susceptibility (loss component) of the sample, $\phi$ is the volume fraction of solid in the suspension, $\rho_{\mathrm{Fe}_{3} \mathrm{O}_{4}}\left(\mathrm{~kg} / \mathrm{m}^{3}\right)$ the density of the magnetite, $f$ is the frequency $(\mathrm{Hz})$ and $H_{0}$ $(\mathrm{A} / \mathrm{m})$ the amplitude of the applied field. Here, we expressed SAR in $(\mathrm{W} / \mathrm{kg})$ in order to use SI units, for clarity purposes; however, a more widespread unit for SAR in the field of hyperthermia is $(\mathrm{W} / \mathrm{g})$, as adopted in the rest of the paper.

\section{B. Properties of the employed sample of colloidal fluid}

Our sample consisted in a vial with a total volume $V_{\text {vial }}=2 \mathrm{~cm}^{3}$, filled with water, agar and magnetite $\left(\mathrm{Fe}_{3} \mathrm{O}_{4}\right)$ nanoparticles. The overall mass of the fluid was $m_{\text {vial }}=1.04 \mathrm{~g}$, thus the density was $\rho_{\text {vial }}=0.52 \mathrm{~g} / \mathrm{cm}^{3}$. The concentration of iron was determined by ICP-OES after acidic digestion of the sample with nitric $(65 \% \mathrm{w} / \mathrm{w})$ and hydrochloric acids (37\%). Consequently, the concentration of $\mathrm{Fe}_{3} \mathrm{O}_{4}$ was calculated on the basis of the stoichiometric ratio between iron and oxygen. Specifically, magnetite nanoparticles were at the $0.12 \%$ of volume fraction ( $\phi=0.0012$ ), whereas the agar was at the $4 \%$. Considering a density for the magnetite of $\rho_{\mathrm{Fe}_{3} \mathrm{O}_{4}}=4.9 \mathrm{~g} / \mathrm{cm}^{3}$, the total magnetite mass can be calculated as: $m_{\mathrm{Fe}_{3} \mathrm{O}_{4}}=\rho_{\mathrm{Fe}_{3} \mathrm{O}_{4}} \times V_{\text {vial }} \times \phi=0.0118 \mathrm{~g}$ (equal to $5.9 \mathrm{mg} / \mathrm{mL}$ ).

Magnetite nanoparticles were organized in the form of nanoclusters; each single nanoparticle presented a mean diameter of $10 \pm 2.6 \mathrm{~nm}$, measured with x-ray diffraction technique (XRD). Precisely, bare magnetite nanoparticles were produced by using the polyol technique. The mean size and mean polydispersity index for the clusters were determined by dynamic light scattering (DLS), and they were found to be $56.9 \pm 0.4 \mathrm{~nm}$ and $0.16 \pm 0.01$, respectively, indicating a narrow size distribution. Transmission electron micrographs (TEM) showed clusters of small numbers of inorganic particles homogeneously dispersed in the polymeric matrix with an average diameter of $50 \mathrm{~nm}$. The clusters are externally coated with a biocompatible polymeric PLGA-PEG shell.

\section{Data acquisition and processing}

The first step in order to apply the proposed method is to acquire a set of SAR measurements in vitro for a colloidal magnetic fluid with nanoparticles at different exposure condition (field amplitude and frequency). We used a commercial heat station (Fives Celes Alu C2 Power Supply, Fivesgroup, France) as an inductive system. The magnetic fluid was contained in a vial placed in the center of the inner volume of a solenoid (Fig. 1).

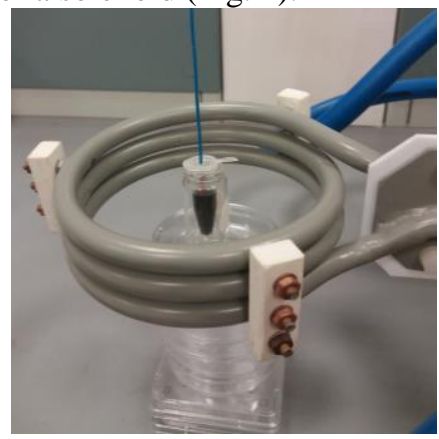

Fig. 1. Experimental set-up for SAR measurements.

We derived SAR measurements (W/g of $\mathrm{Fe}_{3} \mathrm{O}_{4}$ ) from temperature monitoring (recorded every second), through an optical fiber thermometer (CEAM, Vr18CR-PC). We exploited the initial slope $(d T / d t)$ of the heating process:

$$
S A R=\frac{C V}{m_{F_{3} O_{4}}} \frac{d T}{d t}
$$

where $C(\mathrm{~J} / \mathrm{L} / \mathrm{K})$ is the specific heat capacity of the sample, $V(\mathrm{~L})$ is the sample volume, and $m_{\mathrm{Fe}_{3} \mathrm{O}_{4}}(g)$ is the mass of iron oxide in the sample. For the specific heat of sample, we specifically took into account only that of water since this latter has the major contribution with respect to the other components. From (7), we indirectly obtained the values of the imaginary component $\chi$ " for each combination of frequency and amplitude reported in Table I. Considering our experimental set-up, it can be pointed out that the presence of agar suppressed the free movement of nanoparticles and, consequently, Brownian relaxation (high viscosity $\eta$ ). For the last reason, we assumed that only Néel relaxation phenomenon was present. It must be noticed that this is a realistic assumption in a clinical environment [28].

Now, under the assumption that $\chi_{0}$ and $\tau$ are independent from frequency, from two values of $\chi^{\prime \prime}(f)$ at different frequencies (see (3)), it was possible to extract $\chi_{0}$ and $\tau$ through a second order equation system: 


$$
\begin{gathered}
\chi_{0}=\frac{\chi_{1}^{\prime \prime}\left[1+\left(2 \pi f_{1} \tau\right)^{2}\right]}{\left(2 \pi f_{1} \tau\right)} \\
\tau=\sqrt{\frac{\chi_{1}^{\prime \prime} f_{2}-\chi_{2}^{\prime \prime} f_{1}}{4 \pi^{2} f_{1} f_{2}\left(\chi_{2}^{\prime \prime} f_{2}-\chi_{1}^{\prime \prime} f_{1}\right)}}
\end{gathered}
$$

We used all the possible permutations of couples of frequencies available, calculating the mean $\overline{\chi_{0}}$ and $\bar{\tau}$ of the various values of $\chi_{0}$ and $\tau$ obtained. In this way, we were able to construct the curve described in eq. (3).

Then, in order to make the fitting more robust, we optimized $\overline{\chi_{0}}$ and $\bar{\tau}$ through a minimization of the distance between this curve and the experimental loss component data (indirectly derived from eq. (7)) with a least square algorithm. Finally, substituting $\overline{\chi_{0}}$ and $\bar{\tau}$ in equation (2), we computed the real component $\chi^{\prime}(f)$ of complex magnetic susceptibility. We derived the complex magnetic permeability from the equation (11).

$$
\mu_{r}(f)=1+\chi(f)
$$

It must be noticed that the procedure is very rapid and general; it can also be applied to any type of magnetic nanoparticles.

TABLE I

EXPERIMENTAL SAR MEASUREMENTS OBTAINED IN VITRO (PER GRAM OF MAGNETITE)

\begin{tabular}{ccc}
\hline \hline $\begin{array}{c}\text { Field Amplitude } \\
(\mathrm{kA} / \mathrm{m})\end{array}$ & $\begin{array}{c}\text { Field Frequency } \\
(\mathrm{kHz})\end{array}$ & SAR (W/g Fe304 $)$ \\
\hline 9.3 & 103 & 14.8 \\
9.0 & 183 & 30.0 \\
10.7 & 203 & 31.8 \\
7.9 & 254 & 28.5 \\
4.1 & 340 & 24.5 \\
5.7 & 340 & 30.9 \\
7.4 & 340 & 46.4 \\
4.8 & 423 & 21.1 \\
6.1 & 423 & 28.6 \\
7.3 & 423 & 45.9 \\
8.8 & 423 & 71.3 \\
\hline
\end{tabular}

\section{Validation of the analytical method}

In a following step, we replicated the experimental set-up for the SAR measurements with the commercial electromagnetic simulation software Feko, based on the Methods of Moments (MoM) [34]. This allowed the validation of the analytical approach previously described.

We designed a solenoid through a CAD modeling with similar electromagnetic properties than those ones experimentally used (Fig. 2). The solenoid had a variable capacitive load $C$ used as a tuning element for obtaining resonance at each chosen frequency. The feeder of the coil had a voltage amplitude, which permitted to replicate the magnetic field amplitude reached during the experimental SAR measurements (Table I).
A cylinder, placed in the center of the solenoid, modeled the vial, which contained the magnetic fluid. In particular, we used the extracted complex magnetic permeability to model numerically the magnetic colloidal fluid properties.

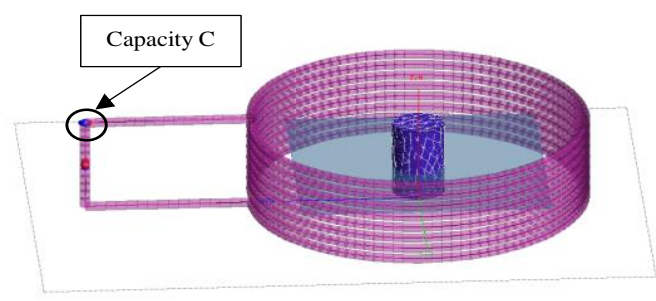

Fig. 2. CAD representation of the experimental set-up.

We performed numerical simulations for each of the operative condition reported in Table I, and we calculated SAR taking into account both electric and magnetic losses, according to (12) ([35]):

$$
S A R=\frac{\sigma_{s}\left|E^{2}\right|}{2 \rho_{\text {vial }}}+\frac{\sigma_{m}\left|H^{2}\right|}{2 \rho_{\text {vial }}}
$$

where $E[\mathrm{~V} / \mathrm{m}]$ and $H[\mathrm{~A} / \mathrm{m}]$ are the amplitude of the electrical and magnetic field in a specific point of the volume of interest respectively, and $\rho_{\text {vial }}$ is the density of the sample contained in the vial. We estimated the electrical and magnetic conductivities, $\sigma_{s}$ and $\sigma_{m}$, as follows [36]:

$$
\begin{gathered}
\sigma_{s}=\omega \varepsilon_{0} \varepsilon_{r} \tan \delta_{s} \\
\sigma_{m}=\omega \mu_{0} \mu_{r} \tan \delta_{m}
\end{gathered}
$$

where $\tan \delta_{s}$ and $\tan \delta_{m}$ are the electric and magnetic loss tangent respectively. $\varepsilon_{r}$ and $\mu_{r}$ are the dielectric relative permeability and the magnetic relative permeability of the sample, respectively. It must be noted that the numerical SAR estimations described in (12) are given per gram of fluid. In order to be compared with the values reported in Table I they must be converted per gram of magnetite.

\section{RESULTS}

Table I contains the SAR measurements obtained by following the procedure described in section II.C. As can be seen from the data, our experimental conditions respected the hypothesis of low amplitude of the magnetic field; therefore, it was theoretically possible to apply the mathematical modeling previously described on our sample of magnetite nanoparticles.

We studied the behavior of the available SAR experimental measurements versus the frequency and versus the amplitude of the magnetic field in order to verify the validity of the Rosensweig's equation (7) for our specific case. In particular, we firstly normalized the SAR data with the square of the amplitude of the magnetic field: in this way, we obtained a new data set dependent only from the frequency. Starting from this new data set, we performed a linear fitting $(f(x)=0.002 x)$. We demonstrated that SAR measurements correctly follow eq. (7) (Fig. 3). 
Then, we replicated the procedure in order to display the dependence of the SAR measurements on the square amplitude of the magnetic field; therefore, we fitted the data with a power law model $\left(f(x)=0.533 x^{2.247}\right)$. Fig. 4 showed the relative log-log plot: SAR measurements at $423 \mathrm{kHz}$ have a dependence with the amplitude of the magnetic field slightly more than quadratic, thus confirming a good agreement with the theoretical model of eq. (7).

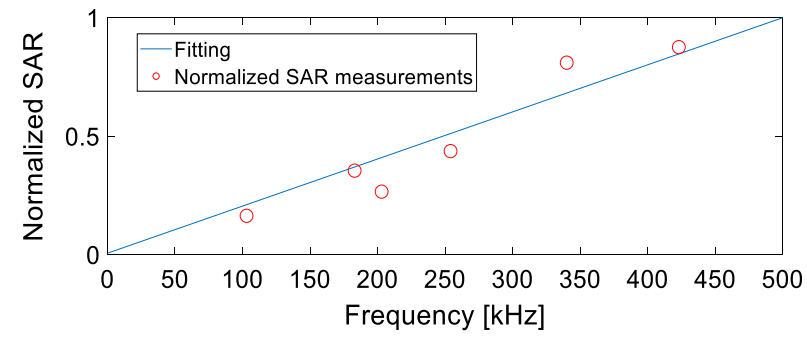

Fig. 3. Linear dependence of the SAR measurements from the frequency.

This analysis confirmed and validated the procedure previously described in order to extract the complex magnetic susceptibility values. The next following step consisted in the derivation of the loss component values (Fig. 5), extracting them from (7) using the experimental SAR values.

Then, we extracted the different $\tau$ and $\chi_{0}$ values from (9) and (10), using the $\chi^{\prime \prime}$ values derived from the experimental SAR measurements, as explained in the previous section. In particular, only valid couples were considered, discarding those which make the argument of eq. (10) negative. It must be noted that the size and the shape of the nanoparticles constituting the sample will present a certain distribution, simply due to production issues. As a consequence, both $\tau_{B}$ and $\tau_{N}$ will vary (eq. (4) and eq. (5)). Nevertheless, as described in the following graphs (Fig. 6), the series of the extracted $\chi_{0}$ and $\tau$ had a small range of variability. Thus, we can assume that the size distribution of the nanoparticles is narrow, confirming the single order Debye relaxation model hypothesis. Afterwards, we performed the mean operation on the two series, obtaining $\overline{\chi_{0}}=0.0076$ and $\bar{\tau}=6.055 \times 10^{-7} \mathrm{~s}$.

Finally, in order to make the fitting more robust, we performed a refinement of $\overline{\chi_{0}}$ and $\bar{\tau}$ minimizing the distance between the analytical curve (described by eq. (3)) and the experimental values (least square algorithm). We found $\bar{\tau}=4.791 \times 10^{-7} s$ and $\overline{\chi_{0}}=0.0067$. The optimized fitting curve with the finally estimated $\bar{\tau}$ and $\overline{\chi_{0}}$ is showed in Fig. 7.

Therefore, we concluded that, for our specific sample of nanoparticles, a RF magnetic field in the range of frequencies around $340 \mathrm{kHz}$ corresponds to the best heating conditions, as could be seen from Fig. (7). Thus, this operative condition could maximize the efficiency of a possible treatment.

In table II, we presented the analytical characterization of the nanoparticles of magnetite, performed with our analytical procedure, in terms of the complex magnetic permeability. These values were calculated using eq. (2), eq. (3) and eq. (11). In this way, we were able to completely characterize the electromagnetic behavior of the magnetic nanoparticles' sample.

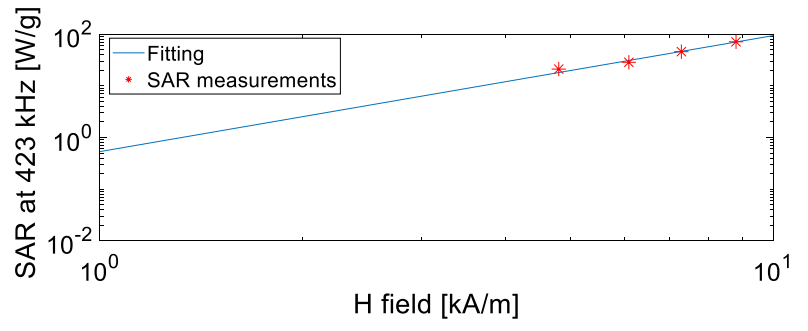

Fig. 4. Quadratic dependence of the SAR measurements from the amplitude of the magnetic field.

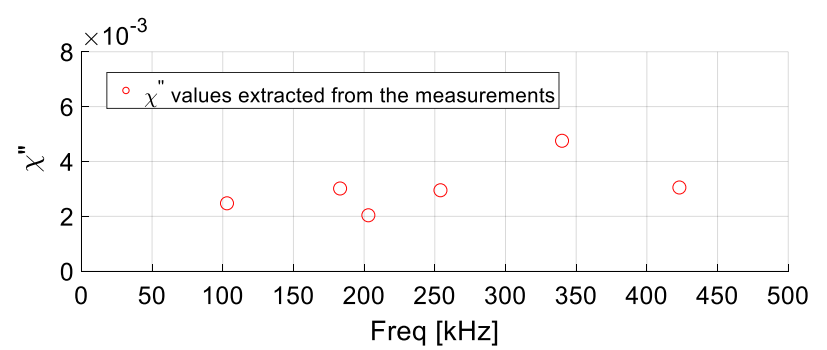

Fig. 5. Imaginary component of complex magnetic susceptibility extracted from inverting (7), averaging for $340 \mathrm{kHz}$ and $423 \mathrm{kHz}$ (multiple acquisitions).

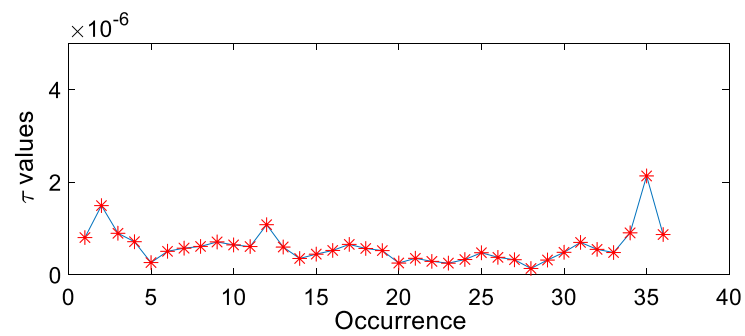

(a)

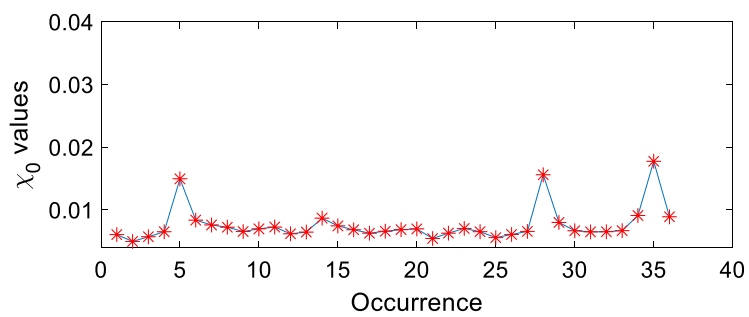

(b)

Fig. 6. $\tau$ (a) and $\chi_{0}$ (b) vectors, extracted from experimental loss component values following the proposed analytical approach.

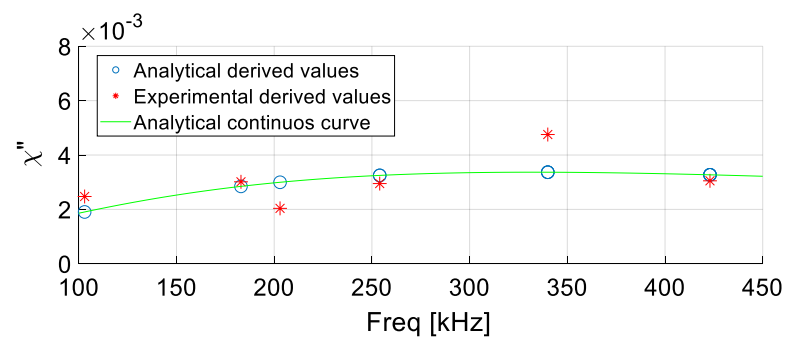

Fig. 7. Comparison between experimentally derived loss component values and the analytical curve derived with our procedure. 
Finally, we performed numerical simulations in order to validate our analytical approach based on the SAR experimental measurements. Fig. 2 described the CAD model; we assigned to the vial the complex magnetic permeability analytically extracted through the model (Table II), and the dielectric properties of the water $\left(\varepsilon_{r}=80\right.$ and $\sigma_{r}=0.6 \mathrm{~S} / \mathrm{m}$ ), correctly following (13) and (14).

TABLE II

COMPLEX PERMEABILITY VALUES EXTRACTED THROUGH THE ANALYTICAL METHOD

\begin{tabular}{ccc}
\hline \hline $\begin{array}{c}\text { Field Frequency } \\
(\mathrm{kHz})\end{array}$ & $\mu_{r}^{\prime}$ & $\mu_{r}^{\prime \prime}$ \\
\hline 103 & 1.0061 & 0.0019 \\
183 & 1.0052 & 0.0028 \\
203 & 1.0049 & 0.0030 \\
254 & 1.0043 & 0.0033 \\
340 & 1.0033 & 0.0034 \\
423 & 1.0026 & 0.0033 \\
\hline
\end{tabular}

In particular, the numerical software, when implementing (12), considers the density of the overall fluid, $\rho_{\text {vial }}$. This means that, in this way, the results would be SAR estimation per gram of fluid. In order to make comparable the experimental SAR (given as W/g of magnetite) and the numerical one, the SAR equation implemented by the software was multiplied by the factor $m_{\text {vial }} / m_{\mathrm{Fe}_{3} \mathrm{O}_{4}}=88.14$.

This is equivalent to assign to the numerical model the effective complex magnetic permeability multiplied by such factor (Table III).

TABLE III

COMPLEX PERMEABILITY VALUES EXTRACTED THROUGH THE ANALYTICAL

\begin{tabular}{ccc}
\multicolumn{2}{c}{ METHOD CONSIDERING THE MASS FACTOR } & $m_{\text {vial }} / m_{\mathrm{Fe}_{3} \mathrm{O}_{4}} \cdot$ \\
\hline \hline $\begin{array}{c}\text { Field Frequency } \\
(\mathrm{kHz})\end{array}$ & $\mu_{r}^{\prime}$ & $\mu_{r}^{\prime \prime}$ \\
\hline 103 & 1.542 & 0.168 \\
183 & 1.456 & 0.251 \\
203 & 1.432 & 0.264 \\
254 & 1.375 & 0.287 \\
340 & 1.290 & 0.297 \\
423 & 1.227 & 0.288 \\
\hline
\end{tabular}

We firstly demonstrated the capability of the software to correctly simulate our experimental set-up, by assigning to the vial the loss component of the complex magnetic susceptibility directly extracted from the experimental SAR values using eq. (7) (Fig. 5), opportunely corrected for the mass factor. Fig. 8 reported the SAR experimental measurements and the SAR values obtained with the simulation, proving an excellent agreement between numerical simulation and experimental measurements. After that, we replicate the simulation using the loss component values estimated with the analytical method, i.e. extracted from (3) through $\overline{\chi_{0}}$ and $\bar{\tau}$, considering the mass factor (Table III). Again, we obtained a satisfying agreement between the experimental results and the analytical modeling, thus validating our proposed approach (Fig. 9).

\section{CONCLUSION}

In this paper, we proposed a general analytical approach to determine the electromagnetic properties of a colloidal magnetic fluid with nanoparticles and agar.

The knowledge of electromagnetic properties is useful to predict the behavior of the nanoparticles at different radiation settings. In particular, this enables to find out the best heating condition in the context of magnetic hyperthermia treatments. In our study, we were able to set the best operative range of frequencies for our specific sample of nanoparticles. It must be pointed out that our method can be a rapid and alternative procedure compared to the directly measurement of magnetic permeability. Moreover, it is possible to apply the method for any type of superparamagnetic nanoparticles and to mimic tissues-like environments exploiting agar properties. Finally, we validated the analytical approach by an electromagnetic simulation software, which is based on the Method of Moments. We successfully replicated the experimental SAR measurements via numerical approach, demonstrating that the analytical extraction of the nanoparticles' magnetic permeability is feasible and effective. The development of standardized methods for the characterization of the nanoparticles, combined with an optimal design of clinical RF coils, can enable the widespread adoption of the magnetic hyperthermia for an efficient cancer treatment. Furthermore, the use of numerical software in this kind of applications will allow efficient and low cost experimental set-up planning for magnetic hyperthermia treatments. To conclude, the numerical approach will also support the development of new and innovative nanostructured hyperthermic agents, opportunely modifying the analytical modeling of the nanoparticles' behavior.

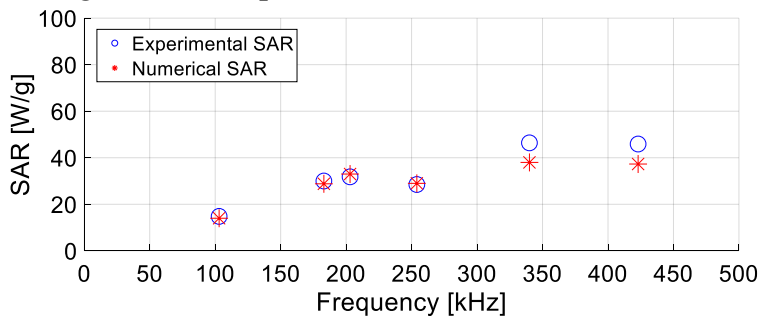

Fig.8 Comparison between numerical and experimentally SAR measurements.

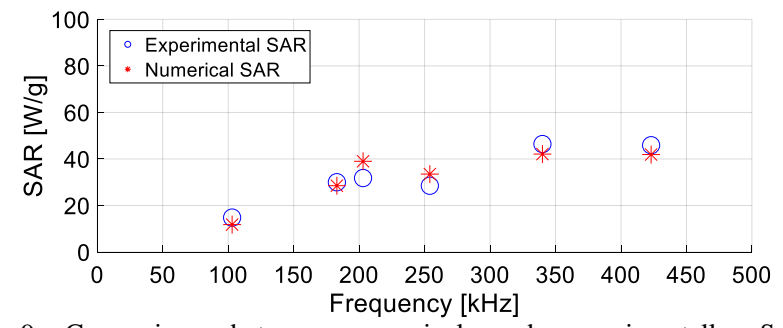

Fig.9 Comparison between numerical and experimentally SAR measurements. 


\section{REFERENCES}

[1] K. M. Krishnan, "Biomedical nanomagnetics: A spin through possibilities in imaging, diagnostics, and therapy," IEEE Trans. Magn., vol. 46, no. 7, pp. 2523-2558, Jul. 2010.

[2] B. Gleich, J. Weizenecker, "Tomographic imaging using the nonlinear response of magnetic particles", Nature Letters, vol. 435, no. 30, Jun. 2005.

[3] J.J. Konkle, P.W. Goodwill, O.M. Carrasco-Zevallos, S.M. Conolly, "Projection Reconstruction Magnetic Particle Imaging", IEEE Trans. Medical Imaging, vol. 32, no. 2, pp. 338-347, Feb. 2013.

[4] V. M. Kulkarni, D. Bodas, D. Dhoble, V. Ghormade, K. Paknikar, "Radio-frequency triggered heating and drug release using doxorubicin-loaded LSMO nanoparticles for bimodal treatment of breast cancer", Colloids and Surfaces B: Biointerfaces, no. 145, pp. 878-890, Elsevier, 2016.

[5] J. Fock, M. Parmvi, M. Strömberg, P. Svedlindh, M. Donolato, M. F. Hansen, "Comparison of optomagnetic and AC susceptibility readouts in a magnetic nanoparticle agglutination assay for detection of C-reactive protein", Biosensors and Bioelectronics, vol. 88, pp. 94-100, 2017.

[6] S. Bae, S. W. Lee, A. Hirukawa, Y. Takemura, Y. H. Jo, S. G. Lee, "AC Magnetic-Field-Induced Heating and Physical Properties of Ferrite Nanoparticles for a Hyperthermia Agent in Medicine", IEEE Transactions on Nanotechnology, vol. 8, no. 1, Jan. 2009.

[7] A. Matsumine, K. Takegami, K. Asanuma, T. Matsubara, T. Nakamura, A. Uchida, A. Sudo, "A novel hyperthermia treatment for bone metastases using magnetic materials", Int. J. Clin. Oncol., vol. 16, pp. 101-108, 2011.

[8] K. Maier-Hauff, R. Rothe, R. Scholz, U. Gneveckow, P. Wust, B. Thiesen, A. Feussner, A. von Deimling, N. Waldoefner, R. Felix, A. Jordan, "Intracranial thermotherapy using magnetic nanoparticles combined with external beam radiotherapy: Results of a feasibility study on patients with glioblastoma multiforme", J. Neurooncol., vol. 81, pp. 53-60, 2007.

[9] P. Wust, U. Gneveckow, M Johannsen, D. Böhmer, T. Henkel, F. Kahmann, J. Sehouli, R. Felix, J. Ricke, A. Jordan, "Magnetic nanoparticles for interstitial thermotherapy - feasibility, tolerance and achieved temperatures", Int. J. Hyperthermia, vol. 22(8), pp. 673-685, Dec. 2006.

[10] M. Johannsen, U. Gneveckow, B. Thiesen, K. Taymoorian, C. H. Cho, N. Waldöfner, R. Scholz, A. Jordan, S. A. Loening, P. Wust, "Thermotherapy of Prostate Cancer Using Magnetic Nanoparticles: Feasibility, Imaging, and Three-Dimensional Temperature Distribution", European Urology, vol. 52, pp. 1653-1662, 2007.

[11] G. Hegyi, G. P. Szigeti, A. Szász, "Hyperthermia versus Oncothermia: Cellular Effects in Complementary Cancer Therapy", Evidence-Based Complementary and Alternative Medicine, 2013.

[12] O. Szasz, A. Szasz, "Oncothermia - Nano-Heating Paradigm", Cancer Sci. Ther., 2014.

[13] N. Cihoric, A. Tsikkinis, G. Van Rhoon, H. Crezee, D. M. Aebersold, S. Bodis, M. Beck, J. Nadobny, V. Budach, P. Wust, P. Ghadjar, "Hyperthermia-related clinical trials on cancer treatment within the ClinicalTrials.gov registry", Int. J. Hyperthermia, vol. 31(6), pp. 609-614, 2015.

[14] R. D. Issels, L. H. Lindner, J. Verweij, P. Wust, P. Reichardt, B. Schem, S. Abdel-Rahman, S. Daugaard, C. Salat, C. Wendtner, Z. Vujaskovic, R. Wessalowski, K. Jauch, H. R. Dürr, F. Ploner, A. Baur-Melnyk, U. Mansmann, W. Hiddemann, J. Blay, P. Hohenberger, "Neo-adjuvant chemotherapy alone or with regional hyperthermia for localised high-risk soft-tissue sarcoma: a randomised phase 3 multicentre study", Lancet Oncol., vol. 11, pp. 561-70, 2010.

[15] R. Di Corato, A. Espinosa, L. Lartigue, M. Tharaud, S. Chat, T. Pellegrino, C. Ménager, F. Gazeau, C. Wilhelm, "Magnetic hyperthermia efficiency in the cellular environment for different nanoparticle designs", Biomaterials, vol. 35, pp. 6400-6411, Elsevier, 2014.

[16] R.E. Rosensweig, "Heating magnetic fluid with alternating magnetic field", in Journal of Magnetism and Magnetic Materials, vol. 252, pp. 370-374, Elsevier, 2002.

[17] M. Hammad, V. Nica, R. Hempelmann, "Synthesis and Characterization of Bi-Magnetic Core/Shell Nanoparticles for Hyperthermia Applications", IEEE Transactions on Magnetics, vol. 53, no. 4, Apr. 2017.
[18] O. M. Bucci, G. Bellizzi, G. G. Bellizzi, "Microwave Broadband Characterization of a Diluted Water-Based Ferrofluid in Presence of a Polarizing Magnetic Field”, IEEE Trans. Magn, vol. 53, no. 3, Mar. 2017.

[19] P.C. Fannin, S.W. Charles, T. Relihan, "On the broadband measurement of the permittivity and magnetic susceptibility of ferrofluids", Journal of Magnetism and Magnetic Materials, no.167, pp. 274-280, Elsevier, 1997.

[20] O.E. Ayala-Valenzuela, J.A. Matutes-Aquino, R. BetancourtGalindo, O. Rodríguez-Fernández, "Complex magnetic susceptibility measurement of a magnetic fluid magnetite based", Int. J. Materials and Product Technology, vol. 27, nos. 1/2, 2006.

[21] I. Hrianca, I. Mălăescu, "The rf magnetic permeability of statically magnetized ferrofluids", Journal of Magnetism and Magnetic Materials, vol. 150, pp. 131- 136, Elsevier, 1995.

[22] O. Ayala-Valenzuela, J. Matutes-Aquino, R. Betancourt-Galindo, L.A. Garcìa-Cerda, O. Rodrìguez Fernàndez, P.C. Fannin, A.T. Giannitsis," Magnetite-cobalt ferrite nanoparticles for kerosenebased magnetic fluids", Journal of Magnetism and Magnetic Materials, vol. 294, Elsevier, 2005.

[23] F. Ahrentorp, A. P. Astalan, C. Jonasson, J. Blomgren, B. Qi, O. T. Mefford, M. Yan, J. Courtois, J. Berret, J. Fresnais, O. Sandre, S. Dutz, R. Müller, C. Johansson, "Sensitive High Frequency AC Suceptometry in Magnetic Nanoparticle Applications", AIP Conf. Proc., 2010.

[24] R. M. Ferguson, A. P. Khandhar, C. Jonasson, J. Blomgren, C. Johansson, K. M. Krishnan, "Size-Dependent Relaxation Properties of Monodisperse Magnetite Nanoparticles Measured Over Seven Decades of Frequency by AC Susceptometry", IEEE Trans. Magn., vol. 49, no. 7, Jul. 2013.

[25] E. Garaio, J. M. Collantes, F. Plazaola, J. A. Garcia, I. CastellanosRubio, "A multifrequency eletromagnetic applicator with an integrated AC magnetometer for magnetic hyperthermia experiments", Meas. Sci. Technol., vol. 25, 2014.

[26] E. Garaio, J. M. Collantes, J. A. Garcia, F. Plazaola, S. Mornet, F. Couillaud, O. Sandre, "A wide-frequency range AC magnetometer to measure the specific absorption rate in nanoparticles for magnetic hyperthermia", Journal of Magnetism and Magnetic Materials, vol. 368, pp. 432-437, Elsevier, 2014.

[27] V. Connord, B. Mehdaoui, R. P. Tan, J. Carrey, M. Respaud, “An air-cooled Litz wire coil for measuring the high frequency hysteresis loops of magnetic samples-A useful setup for magnetic hyperthermia applications", Review of Scientific Instruments, vol. 85, 2014.

[28] S. Dutz, M. Kettering, I. Hilger, R. Müller, M. Zeisberger, "Magnetic multicore nanoparticles for hyperthermia-influence of particle immobilization in tumour tissue on magnetic properties", Nanotechnology, vol. 22, IOP Publishing, 2011.

[29] K. Iizuka, "An Agar-Agar Chamber for Study of Electromagnetic Waves in an Inhomogeneous Medium", IEEE Transactions on antennas and propagation, vol. ap-19, no. 3, Mar. 1971.

[30] P.C. Fannin, L. Cohen-Tannoudji, E. Bertrand, A.T. Giannitsis, C. Mac Oireachtaigh, J. Bibette, "Investigation of the complex susceptibility of magnetic beads containing maghemite nanoparticles", in Journal of Magnetism and Magnetic Materials, vol. 303, pp. 147-152, Elsevier, 2006.

[31] R. Hergt, S. Dutz, M. Zeisberger, "Validity limits of the Neeel relaxation model of magnetic nanoparticles for hyperthermia", Nanotechnology, vol. 21, IOP Publishing, 2010.

[32] S. Dutz, R. Hergt, "Magnetic particle hyperthermia-a promising tumour therapy?" Nanotechnology, vol. 25, IOP Publishing, 2014.

[33] W. F. Brown, "Thermal fluctuations of a single-domain particle", Physical Review, vol. 130, no. 5, 1963.

[34] J. Volakis, K. Sertel, "Integral equation methods for electromagnetics", The Institution of Engineering and Technology, 2012.

[35] Dong-Yeol Lee, Yu-Ri Lee, Young-Jun Ju, Yoon-Myoung Gimm, "Numerical analysis of thermal effect of the lossy magnetic materials in the human model by high frequency magnetic field", International Symposium on Antennas and Propagation, Oct. 2011.

[36] F. J. German, G. K. Gothard, L. S. Riggs, "Modeling of materials with electric and magnetic losses with the symmetrical condensed TLM method", IEEE Electronic Letters, vol. 26, no. 16, August 1990. 


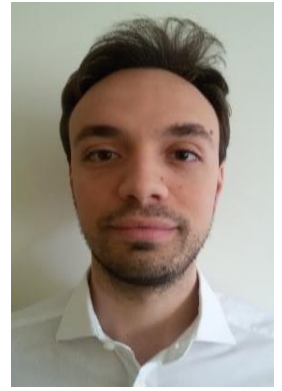

Danilo Brizi, St.M'17, (Viterbo, Italy, 1992) received the M.S. Laurea degree (summa cum laude) in Biomedical Engineering from the University of Pisa in 2016. Currently, he is a Ph.D. student in Information Engineering at the University of Pisa. His research interests include hyperthermia with magnetic nanoparticles, MRI filter design and Wireless Power Transfer applications.

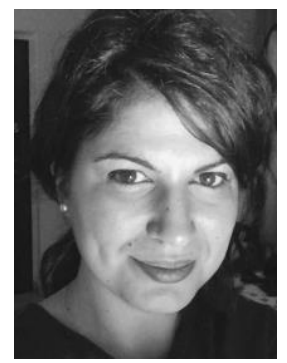

Nunzia Fontana (Agrigento, Italy, 1984) received the Master degree in Telecommunications Engineering (summa cum laude) from University of Pisa (UniPi, Italy) in 2008 where she conducted her Ph.D. in Remote Sensing from 2009 to 2011. In 2011 she carried out an internship at Queen Mary, University of London. From 2012 to 2016 she was Post-doc researcher at the UniPi. She worked at UniPi as teaching assistant from 2010 to 2015 in the courses of Telecommunications and Biomedical Engineering. Since 2016 she is Researcher at CNIT (Italian InterUniversity Consortium for Telecommunications) and she works as external lecturer in the course of Biomedical Engineering, UniPi. Her main research topics include: antenna design, prototyping and RF testing; impedance matching networks design, prototyping and RF testing; Radio Frequency coils design for Magnetic Resonance and RF testing; bioelectromagnetics. The results of her research activity have been currently published on several international scientific journals and on a number of international conference proceedings.

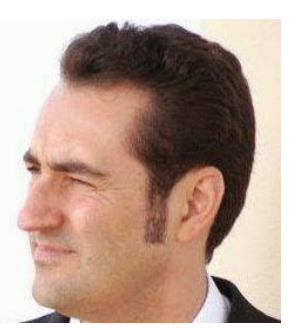

Giulio Giovannetti received the Master's degree in Electronic Engineering, the Master's degree in Biomedical Engineering and the $\mathrm{PhD}$ degree in Automatics, Robotics and Bioengineering from the University of Pisa, Italy, in 2000, 2004 and 2008, respectively. Since 2009 he is a Researcher at C.N.R. Institute of Clinical Physiology in Pisa and from 2012 to 2015 he was Adjunct Professor at Engineering School (Biomedical Engineering) of Pisa University (Italy).

His research interests include electromagnetic field simulations and measurements in Magnetic Resonance environment, radiofrequency coil design and simulation for Magnetic Resonance Imaging and Spectroscopy, dosimetry and power deposition simulations and measurements.

He has published 140 among international journals, conference papers and book chapters on topics related to his research themes.

$\mathrm{He}$ is a reviewer for some scientific international journals and he has got collaborations with some high-tech industries involved in different fields of research and development.

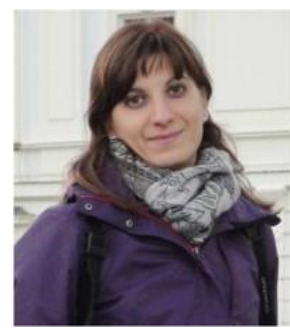

Alessandra Flori obtained her MSc degree in Applied Physics at the University of Pisa (Italy), with background in Medical Physics, and obtained her PhD in "Novel Strategies in Biomedical Research" at the Institute of Life Sciences of Scuola Superiore Sant'Anna (Pisa, Italy). Her research activity focuses on the application of ${ }^{13} \mathrm{C}$-labeled hyperpolarized contrast media for metabolic studies in vivo by Magnetic Resonance Spectroscopy. Part of her research activity deals with the evaluation and the study of the relaxation profile of nanoparticles, used as Magnetic Resonance Imaging contrast media for biomedical applications.

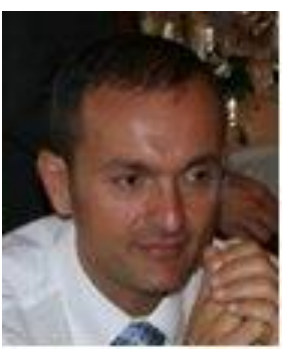

Luca Menichetti: Starting from a background in radiochemistry of Transition Metals at JRCInstitute for Transuranium Elements (Karlsruhe, Germany), Luca Menichetti (LM) moved his interests in the field of tracer chemistry for medical applications for the development of molecular imaging techniques at National Research Council Institute of Clinical Physiology (CNR IFC) in Pisa (Italy). The know-how in tracer-chemistry has been extended to different imaging modalities, such as Magnetic Resonance Spectroscopy exploiting the hyperpolarization of 13C-molecules and the development of novel multimodality based on nanostructured multifunctional materials. Since 2013 LM is contract Professor at the Department of Chemistry at University of Pisa and the Department of Pharmacy at University of Siena: LM is the scientific coordinator of national projects in the field of nanomedicine and is working in the frame of national and international projects and is author of more than ISI 55 publication.

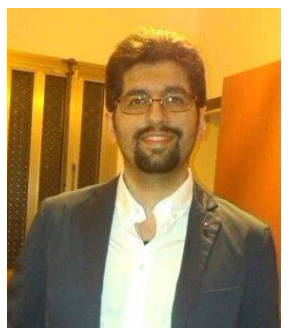

Dr. Saer Doumett, PhD: studied Chemistry at University of Damascus (Syria) and got his degree in 2003. In 2007 he got his $\mathrm{PhD}$ in Chemical Sciences with a thesis on wastewater treatment and contaminated soil remediation. In Colorobbia company since 2012, he works in the nanomedicine division in Colorobbia Research Center (Ce.Ri.Col) and he is coowner of six international publications in the field of nanomedicine.

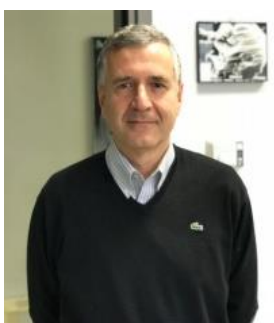

Dr. Giovanni Baldi, PhD: is Director of Colorobbia Research Center (Ce.Ri.Col) 1999present, he is also Scientific Director of NAnott Research Laboratory of Tuscan Nanotechnology from 2012. He is in the Steering Commitee of Nanotech.it Society and in the board of Italian Society of Nanotoxicology. IP project evaluator for the 7th European Framework Programme. He is owner of 24 PCT patents and 95 publications in the field of glass, ceramics, glass-ceramics, functional coatings, nanostructured surfaces, synthesis of nano-structured particles, nanobiomaterials and drug delivery.

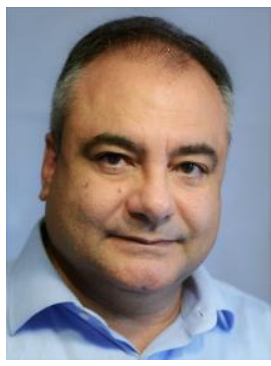

Agostino Monorchio (S'89-M'96-SM'04F'12), Professor at the Department of Information Engineering of the University of Pisa, is active in several areas related to electromagnetics, including computational numerical techniques, microwave metamaterials, radio propagation for wireless systems, the design and miniaturization of antennas and electromagnetic compatibility, microwaves biomedical applications.

His activity is mainly carried out at the Microwave and Radiation Laboratory (www.mrlab.it) of the same Department, together with a large group of PhD students, Post-Docs and research associates. 